\title{
URBAN ARTEFACT, Protocell for the activation of abandoned public spaces in degraded neighborhoods
}

\author{
Sandra Yunuén Heredia Balcázar \\ Universidad Michoacana de San Nicolás de Hidalgo | México | herediab.sandra@gmail.com \\ Juan Carlos Lobato Valdespino \\ Universidad Michoacana de San Nicolás de Hidalgo | México | jlobato@umich.mx
}

Jorge Humberto Flores Romero

Universidad Michoacana de San Nicolás de Hidalgo | México | jhfloresr@gmail.com

\begin{abstract}
The work addresses the problem of the reactivation of abandoned or underused public spaces in degraded neighborhoods. It proposes for this the development and implementation of an urban artifact whose metaphor is the protocell. With the development of bottom-up mobile system protocols, it aims to influence a process of urban regeneration in marginalized neighborhoods. The case study is located in a peripheral area of the city of Morelia, Michoacán, México. Designing, exploring and testing a textile manufacturing artifact with a hybrid process of digital and analog technology, a performance installation that responds to the physical, social and human environment.
\end{abstract}

Keywords: Design; Emerging; Innovation; Social; Public.

\section{INTRODUCCIÓN}

El proceso del diseño en el mundo contemporáneo se caracteriza por buscar soluciones a problemas complejos, tomando en consideración los contextos sociales y culturales, en búsqueda de una mejor calidad de vida. La convergencia de tecnología y materiales en un contexto social con el propósito de mejorar las condiciones de vida, son ahora competencia que se buscan y se promueven en el diseñador extendiéndose a las ciencias del comportamiento y la comprensión antropológica cultural (Manu, 1998).

El trabajo que aquí se presenta propone que mediante el diseño y la construcción de un artefacto urbano móvil e interactivo, se implante un modelo experimental que permita la correlación de forma diferente con el entorno, con la acción humana y la colectividad de la ciudad, especialmente en aquellas zonas con espacios subutilizados donde el artefacto puede funcionar como un laboratorio emergente que permita explorar diversas formas de interacción, convirtiéndolas así en áreas de recreación pública donde se provoque vida pública que ayude a revitalizar la vida urbana por medio de la participación y la inclusión.

Las ciudades se encuentran en constante crecimiento y cada vez son más las personas que las habitan, de acuerdo a la ONU el $55 \%$ de las personas del mundo viven en ciudades y se estima que para el año 2050 éste número aumentará en un $13 \%$ (ONU, 2018), sin embargo muchas de ellas y en especial las que se encuentran en los países en desarrollo presentan problemáticas complejas en diversos ámbitos, el espacio público es uno de ellos, al ser un componente vital de la ciudad éste influye directamente en la calidad de vida de los ciudadanos.
El espacio público es el patio de las ciudades, el sitio donde se ejercitan la convivencia, la interacción y la integración social, su distribución así como los elementos que lo componen generan grandes impactos en la transformación de las dinámicas urbanas, ya que ofrecen un espacio idóneo para el sano esparcimiento y la recreación (Gehl \& Gemzøe, 2002), sin embargo muchos de los espacios enfocados en éstas actividades lúdicas y deportivas se encuentran subutilizados y en un estado de degradación; estos sitios son espacios públicos de barrio (SEDESOL, 2010) a los cuáles se puede acceder a pie de manera cotidiana y tienen dimensiones pequeñas en comparación con los espacios públicos monumentales de las ciudades, como pueden ser plazas públicas o unidades deportivas.

El abandono de estos espacios ha sido consecuencia de diversas causas que se han dado a lo largo del tiempo y a la par con el crecimiento acelerado de la ciudad, en el caso de Morelia la cual se aborda como caso de estudio, se observó un crecimiento acelerado a partir del año 1990 (IMPLAN, 2016) en el cual la población se comenzó a extender hacia la periferia y los espacios públicos recreativos fueron insuficientes para la población, a raíz de haberse detectado un déficit de área verdes y espacios recreativos se comenzó a construir infraestructura deportiva básica en las colonias con índices de marginación; actualmente es común encontrar un gran número de planchas de concreto con medidas mínimas para alojar una cancha de basquetbol.

Actualmente no existe un registro preciso de la cantidad ni del estado precario de estas canchas públicas, lo que evidencia su invisibilidad dentro de la planificación urbana, sin embargo, es usual encontrarlas en todo el país, con 
características similares, una imagen deteriorada y baja permanencia, lo cual los convierte en espacios idóneos para el vandalismo, estos sitios se han convertido también en una herramienta política para mostrar interés por el bienestar social, sin embargo, hay poco estudio y seguimiento detrás de la implementación de esta infraestructura.

Como parte del reconocimiento de la relevancia que tiene el espacio público en las ciudades, las agendas urbanas han realizado diversos estudios y esfuerzos para recuperar áreas en condiciones degradadas en la última década, en la ciudad de Morelia el índice de Ciudad Próspera (CPI) ha identificado que es necesario fortalecer las políticas del ámbito urbano $(\mathrm{ONU}, 2015)$ en los 5 últimos años se han realizado diversas campañas de recuperación de este tipo de canchas a nivel local ya que se ha detectado el abandono por parte de las políticas públicas en el mantenimiento de la infraestructura recreativa y deportiva de la ciudad de Morelia, Michoacán, México (Ojeda, 2019) Sin embargo el estado en que se encuentran estos sitios sigue siendo de poco uso e indiferencia por parte de los habitantes de las colonias en las que se encuentran, de las cuáles se han podido identificar al menos 23 en la ciudad de Morelia.

El efecto negativo que producen las canchas subutilizadas es contrario a su potencial recreativo y de encuentro, reflejando los diversos conflictos y ruptura del tejido social, así como la baja interacción y sentido de pertenencia con el espacio público inmediato, esto ha provocado que se vea disminuida la capacidad del espacio colectivo para generar relaciones humanas activas o de permanencia. (Borja et al., 2000)

Dado el potencial poco explotado que tienen estos espacios para el mejoramiento de la calidad de vida de los ciudadanos es pertinente implementar artefactos que permitan establecer un diálogo con los habitantes de una comunidad y detonar actividad y encuentro, la intervención de las canchas deberá permitir explorar el espacio desde una nueva perspectiva, donde se integre la opinión y la interacción social a la toma de decisiones, se genere una mayor atracción e identidad entre espacio recreativo y habitante, y se aprovechen las oportunidades que brinda el diseño, la tecnología y la innovación social al desarrollo de respuestas enfocadas en las necesidades de las personas. (Manzini \& Vega Pindado, 2015)

\section{OBJETIVO GENERAL}

El tema de investigación tiene como objetivo principal diseñar e implementar un artefacto urbano emergente, ligero e interactivo, el cual plantee estrategias que estimulen el incremento de las dinámicas sociales positivas en las canchas públicas subutilizadas y generen condiciones de empoderamiento del espacio público entre los habitantes de una comunidad.

\section{OBJETIVOS PARTICULARES}

1. Identificar y mapear canchas públicas destinadas al deporte y la recreación en condiciones subutilizadas y deterioradas, pertenecientes a grupos sociales consolidados, unidos y participativos.
2. Seleccionar el o los casos de estudio y analizar las dinámicas sociales de la comunidad.

3. Diseñar y fabricar un artefacto urbano emergente, ligero y adaptable a diversas estrategias de activación del sitio.

4. Implementar el artefacto en el sitio de estudio y medir su impacto de acuerdo a indicadores de afluencia y calidad del espacio público

5. Analizar los datos obtenidos durante la intervención y contrastar con los objetivos planteados

6. Plantear conclusiones y proponer posibles mejoras tanto en el artefacto como en el sitio de estudio.

\section{METODOLOGÍA}

Se plantea una metodología versátil para invitar a las personas a explorar el potencial futuro del momento presente a través de una serie de perspectivas que deben tenerse en cuenta si queremos dirigir nuestro rumbo sabiamente hacia un futuro impredecible. El marco de trabajo "Tres Horizontes Tecnológicos" es una herramienta de previsión que puede ayudarnos a estructurar nuestro pensamiento sobre el futuro de manera que genere innovación. Tecnología de Construcción Política (TCP), Tecnología de Construcción Social (TCS), Tecnología de Construcción Digital (TCD).

El marco nos ayuda a ser más conscientes de cómo nuestras intenciones, comportamientos individuales y colectivos configuran activamente el futuro de hoy. Al mapear tres formas de relacionarse con el futuro desde la perspectiva de los tres horizontes (Wahl, 2016), podemos aportar el valor de cada uno de ellos a la conversación de una manera generativa que fomente la comprensión y la conciencia futura como la base para la acción colaborativa y la innovación transformadora.

La metodología aplicada también incorpora la experimentación y la iteración de los procesos que se dan dentro de los tres horizontes, dando cabida a la revisión de diversas posibilidades que puede tener el proyecto y su desarrollo no lineal, lo cual permite que al detectar vacíos en cualquier parte del proceso se pueda retomar dicho punto y realizar correcciones.

El desarrollo del artefacto urbano propuesto se compone de 6 fases que no son necesariamente consecutivas, pero cuya exploración nutre una a la otra, permitiendo enriquecer el proceso y generar una propuesta que cumpla con los objetivos, las etapas son:

1.-Reconocimiento de las canchas subutilizadas a intervenir: el punto de partida de este trabajo es la identificación de los sitios potenciales que reúnen las condiciones planteadas dentro del problema de investigación.

2.-Mapeo de información: Posterior a una exhaustiva revisión de datos estadísticos y levantamiento en campo se vacía la información básica del sitio a intervenir 
3.- Diseño del artefacto: De acuerdo con las características detectadas en las canchas subutilizadas y las necesidades de la población del caso de estudio se realiza un diagnóstico que permite definir los criterios y herramientas de diseño

4.-Fabricación del artefacto: fase de materialización del diseño definido

5.-Implementación del artefacto: intervención de sitio y desarrollo de actividades detonadoras

6.-Evaluación y retroalimentación: evaluación de posibles mejoras del artefacto, análisis y reflexión de datos obtenidos

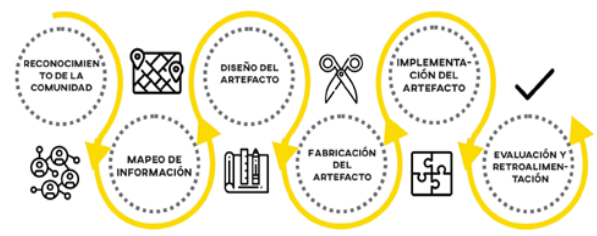

\section{CASO DE ESTUDIO}

El caso de estudio se localiza en la colonia de alta marginación José María Pino Suárez, en la ciudad de morelia, Michoacán, en la cual se ubica una cancha recreativa con baja actividad y escasa apropiación por parte de la población

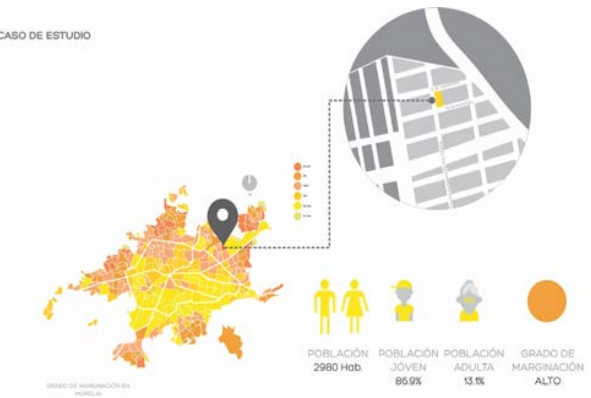

Figura 2: Localización y características del caso de estudio

La población y el sitio de estudio reúnen ciertas características relevantes que hacen factible la experimentación piloto en este sitio, como lo es la cantidad de habitantes jóvenes que son aquellos que requieren de espacios y actividades recreativas públicas; el grado alto de marginación en el barrio, lo cual demandas nuevas políticas que favorezcan espacios gratuitos de sana convivencia y el potencial que tiene el sitio al ser el único espacio con esta vocación en un radio de $400 \mathrm{mts}$.

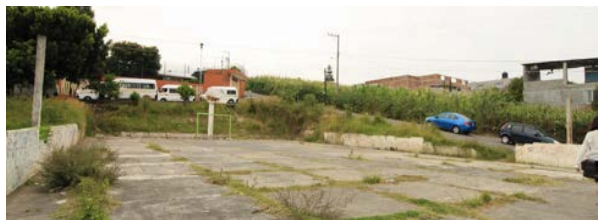

Figura 3: Cancha recreativa para caso de estudio
La población muestra fue analizada previamente mediante encuestas y talleres participativos, lo cual permitió obtener información acerca de las actividades realizadas y deseables, conocer estas necesidades es parte primordial para el diseño del artefacto, el cual tiene como base la participación social en el proceso de transformación.

De esta manera se pretende que el diseño generado y la comunidad creen un "bridge" entre el habitante y el artefacto, por lo cual es tan relevante conocer sus necesidades y tomar en cuenta sus visiones, asegurarnos de generar estas conexiones en el desarrollo del proyecto da solidez al proceso de la innovación social para los escenarios urbanos actuales («Ezio Manzini», 2012).

\begin{tabular}{|l|}
\hline \multicolumn{1}{|c|}{ CALIDAD DEL ESPACIO PÚBLICO } \\
\hline IDEALES \\
\hline $\begin{array}{l}\text { Actividades } \\
\text { necesarias }\end{array}$ \\
\hline $\begin{array}{l}\text { Actividades } \\
\text { opcionales }\end{array}$ \\
\hline $\begin{array}{l}\text { Actividades } \\
\text { resultantes } \\
\text { (sociales) }\end{array}$ \\
\hline
\end{tabular}

Figura 4: Calidad del espacio público

El caso de estudio refleja una necesidad de aplicar estrategias de revitalización que estimulen la actividad en la cancha y propicien la atracción y el encuentro.

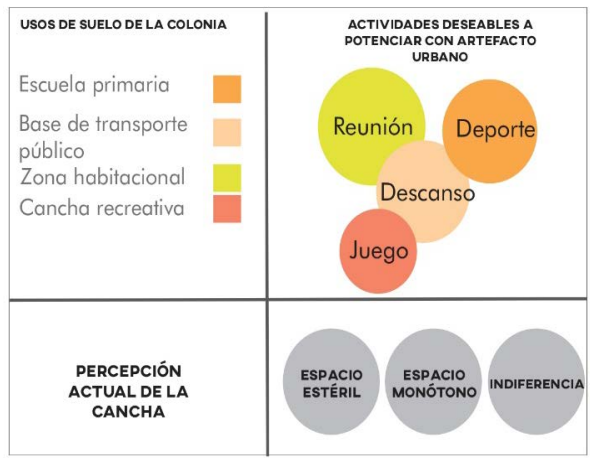

Figura 5: Características potenciales del caso de estudio

En torno a las condiciones expuestas anteriormente, la investigación plantea la siguiente pregunta ¿Cómo puede un artefacto urbano catalizar el incremento de actividades opcionales y resultantes en el sitio y generar atracción y sentido de pertenencia en las personas? Para lo cual se plantea el artefacto "Célula Urbana" cuya función es detonar el encuentro en la cancha y poder medir el impacto que tiene el aumento de actividades de sana recreación en un sitio subutilizado y generalmente ignorado. 


\section{TÉCNICAS E INSTRUMENTOS}

\section{"CÉLULA URBANA"}

Derivado del análisis de la comunidad y del sitio surgen tres objetivos básicos sobre los que se dirige el diseño de la unidad de vida "célula urbana" los cuáles son detonar encuentro, estimular pertenencia e incentivar cooperación. La célula busca adherirse temporalmente en el sitio e integrarse como parte del tejido urbano y por medio de su estructura cumplir con estas tres funciones sociales que incrementen los usos del espacio, visibilicen el sitio y simulen vida dentro de la cancha, re activando el espacio no solo de forma física sino también en la percepción de los habitantes de la comunidad. "Las protoceldas son la etapa de transición hacia la creación de células completamente artificiales usando un enfoque de abajo hacia arriba para su montaje, y son una parte esencial del descubrimiento de procesos vivos en lugar de la meta."(Spiller, 2011)

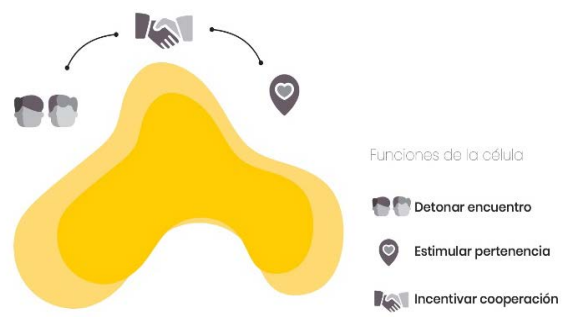

Figura 6: Diagrama de funcionamiento de la célula

El diseño del artefacto utiliza como herramienta la experimentación analógica combinada con diseño y fabricación digital, el uso de software asistido por computadora y especializado en modelar elementos orgánicos permite explorar variaciones y simulaciones en un artefacto que simula una estructura viva que forma parte del organismo que es la ciudad y que genera un contraste con el ambiente estéril en el que se inserta.

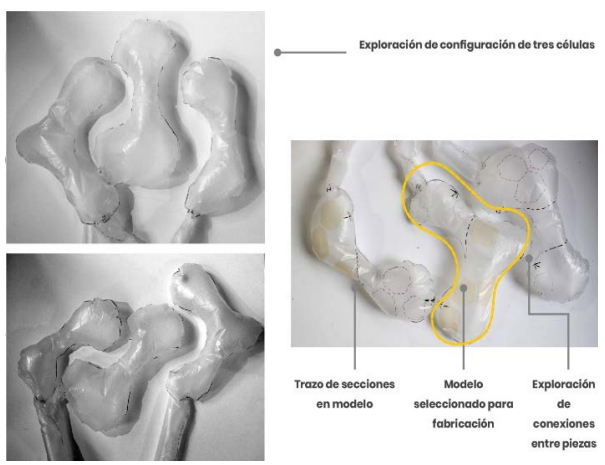

Figura 7: Prototipado y pruebas de funcionamiento
Sobre la membrana plástica utilizada para la fabricación del prototipo se trazan las áreas destinadas a ser sólidas y translúcidas, con el fin de generar un espacio interior que proporcione una experiencia sensorial y permita captar fragmentos del entorno para la observación del mismo

Tabla 1: Características de la muestra de prototipado

\begin{tabular}{|l|l|}
\hline \multicolumn{2}{|c|}{ Primeras muestras de prototipado } \\
\hline Material de Experimentación & $\begin{array}{l}\text { Película de polietileno de alta } \\
\text { densidad }\end{array}$ \\
\hline Grosor & $0.014 \mathrm{~mm}$ \\
\hline Técnica de unión de pieza & Soldado térmico \\
\hline Motor de inflado & Ventilador de 7" \\
\hline
\end{tabular}

\section{PROCESO DE DISEÑO ASISTIDO CON SOFTWARE DIGITAL}

Habiendo elegido el elemento a prototipar y probar se hace uso de las herramientas del software de modelado que permitirá realizar los procesos de fabricación de manera más eficiente, así como la utilización de una estructura ligera que pueda ser transportada por distintos casos de estudio a largo plazo y probada en diversas condiciones físicas y sociales.

Esta combinación de técnicas de experimentación analógicas y digitales para el diseño de un artefacto facilita el entendimiento de la misma manera tangible y permite hacer uso de recursos accesibles y coherentes dentro del contexto latinoamericano.

El prototipo se explora a partir de la geometría anteriormente planteada en base al diagrama de funcionamiento, la cual se digitaliza para formar una estructura sobre la cual sea posible correr una simulación de presión interna y explorar las dimensiones que permitan la habitabilidad, de ésta simulación se obtiene un elemento tridimensional con una altura interna de $1.80 \mathrm{~m}$ y un ancho de $6.50 \mathrm{~m}$, lo cual permite el encuentro y la interacción dentro de la estructura; con la finalidad de crear un elemento que tenga una experiencia más dinámica se modifica la altura constante al comprimir la estructura en los tramos más delgados, de ésta forma el recorrido presenta obliga a cambiar de posición para para pasar de un extremo a otro.

La fabricación del elemento se realiza a partir de la generación de polígonos en la membrana, los cuales tienen el fin de funcionar como entradas de luz y a la vez enmarcan el entorno en fragmentos que permiten observar con mayor detenimiento el espacio y generar una interacción entre el exterior y el interior. A partir de dicho criterio se procede a realizar el patronaje de los polígonos, con el objetivo de agilizar la fabricación se divide la célula en tres secciones generales y sus polígonos aislados. 


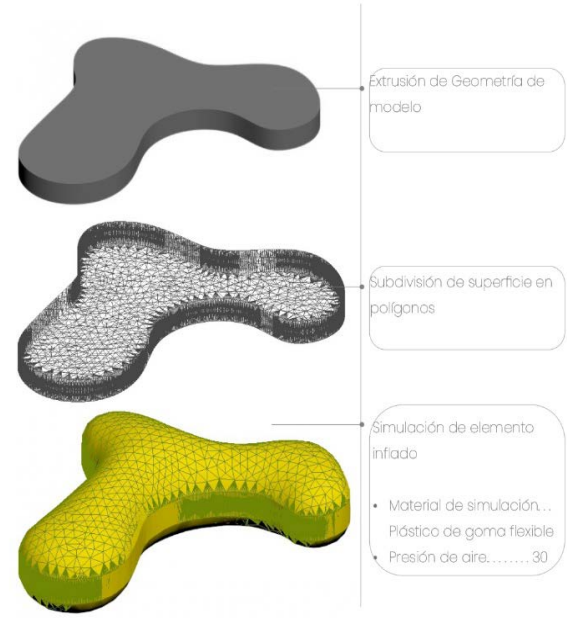

Figura 8: Simulación de membrana textil con presión interna

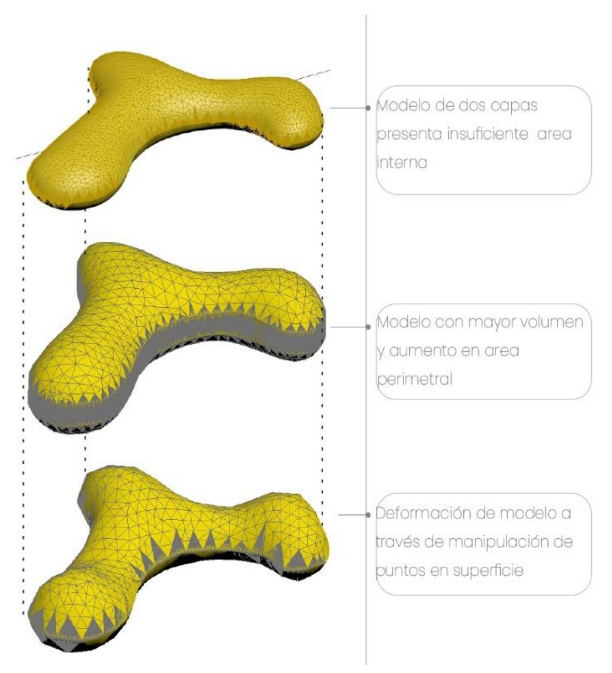

Figura 9: Manipulación geométrica de membrana

Estas simulaciones digitales amplían la posibilidad de manipular el elemento de manera ágil y concreta, lo que ayuda a acortar el tiempo de fabricación y proporciona información certera del elemento.

Tabla 2: Características de la muestra de simulación digital

\section{Simulación de membrana con presión interna de aire}

\begin{tabular}{|l|l|}
\hline Software de experimentación & Autodesk 3D Max \\
\hline Material de simulación & Plástico flexible \\
\hline Nivel de tensión & 30 \\
\hline
\end{tabular}

A partir de éste modelo obtenido se procedió a generar el patronaje digital, este proceso permite replicar el elemento y modificarlo las veces necesarias con el fin de mejorar la experiencia de las personas, posterior a la generación de la superficie en $2 \mathrm{D}$ se procedió a fabricar el prototipo a escala a base de película plástica y unión con cinta entre las piezas, las cuales están etiquetadas con su código correspondiente para guiar el armado, el inflado de la pieza fue exitoso ya que se obtuvo la geometría planteada, la estructura presentó condiciones aptas para la habitabilidad y para funcionar como un elemento de atracción al sitio, ya que la presiona interna permite que se sostenga en posición vertical, con ayuda de contrapesos.

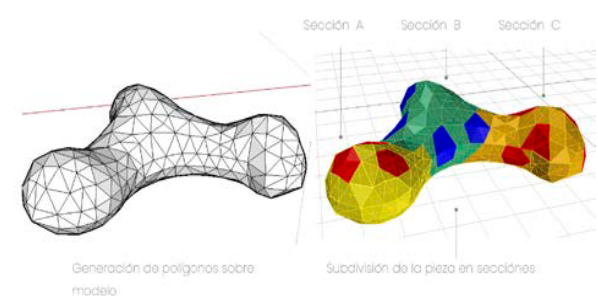

Figura 10: Subdivisión del elemento y delimitación de patrones

El prototipo fabricado a partir del patronaje propuesto facilita delimitar los polígonos en los cuáles se requiere un cambio de material o color, así como la obtención de tramos más grandes de patronaje al generar grupos de polígonos que pueden ser desplegados de manera conjunta; este prototipo es capaz de ser re escalado y replicado en otros materiales similares.

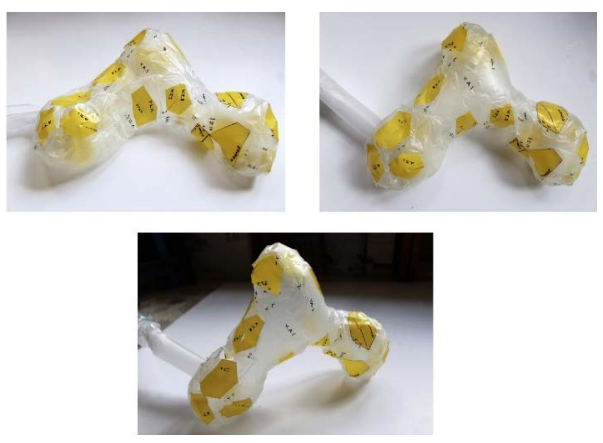

Figura 11: Prototipo a escala de célula

Tabla 3: Características de la muestra de simulación digital

\begin{tabular}{|l|l|}
\hline \multicolumn{2}{|c|}{ Prototipo a base de patronaje } \\
\hline Software de patronaje & Rhinoceros 3D \\
\hline Técnica de patronaje & Desarrollo 2d de superficies \\
\hline Material de prototipado & Plástico de alta densidad \\
\hline
\end{tabular}




\section{RESULTADOS}

Se propone realizar los experimentos haciendo uso de estructuras neumáticas, diseñadas con una serie de elementos interactivos, ligeros y fáciles de manipular, a través de los cuáles se provoque la exploración sensorial y se invite a la expresión de ideas por parte de la comunidad, con la finalidad de incentivar a la participación ciudadana, incrementar el sentido de pertenencia y reimaginar el potencial de las áreas subutilizadas que son punto de encuentro, un espacio que al aprovecharlo los habitantes pueden ser capaces de descubrir que cuentan con áreas desaprovechadas y que ellos tienen la capacidad y el derecho de re habitarlas.

La estructura de la célula urbana se compone de tres elementos principales; membrana exterior, la cual será la contenedora de los elementos y las actividades potenciadoras, el núcleo interior que permitirá hacer habitable ésta estructura neumática y las actividades potenciadoras que proponen una serie de dinámicas de interacción con el sitio y entre las personas.

Se plantea que las dinámicas en el interior de la estructura se den de manera espontánea y las personas utilicen su creatividad para activar el espacio con actividades como el juego, la convivencia y el descanso.

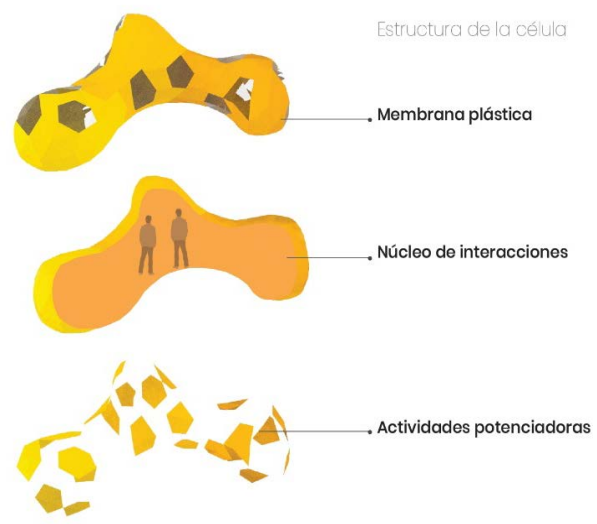

Figura 12: Diagrama de estructura celular

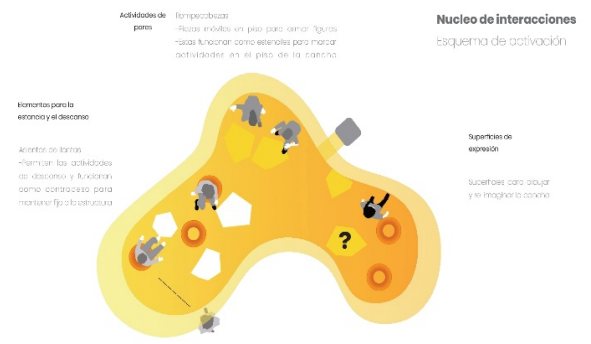

Figura 13: Diagrama de funcionamiento e interacciones

\section{DINÁMICAS DE ACTIVACIÓN}

Como parte de las dinámicas que la membrana puede albergar en su interior por si sola, se integran además una serie de dinámicas provocadoras de actividad y reconocimiento del sitio, las cuales se integran a la membrana de maneral desmontable en forma de láminas que corresponden formalmente con algunos de los polígonos aislados que fueron asignados para tener un cambio de material translúcido y funcionar como entradas de luz.

Dichos activadores contienen interrogantes relacionadas con el espacio recreativo, su percepción y mejoramiento, esos pueden ser manipulados por la comunidad y funcionar como superficie de expresión de opiniones a través del dibujo.
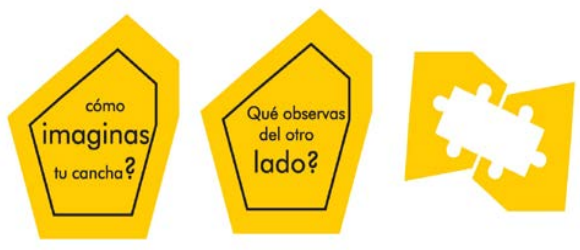

$$
\begin{gathered}
\text { INVITAR } \\
\text {-A la interacción con } \\
\text { el ortéfocto y el } \\
\text { entomo }
\end{gathered}
$$
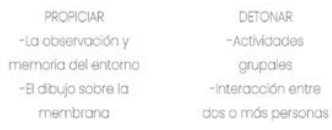

Figura 14: Diagrama de activadores

\section{EXPERIMENTACIÓN CON PROTOTIPO A ESCALA 1:1}

El proceso de fabricación del prototipo a una escala mayor, permite la experimentación en sitio; a partir del patronaje obtenido en las etapas previas de experimentación digital se procede a fabricar la membrana en textil impermeable y plástico calibre 400 , estos materiales contienen el aire dentro de la membrana, aportan ligereza, un tamaño reducido al estar compactado y uno amplio al estar en funcionamiento, alcanzando un tamaño de 7 metros de largo y 1.60 metros de alto. Las uniones de las piezas se realizan por medio de costura en máquina de coser industrial.

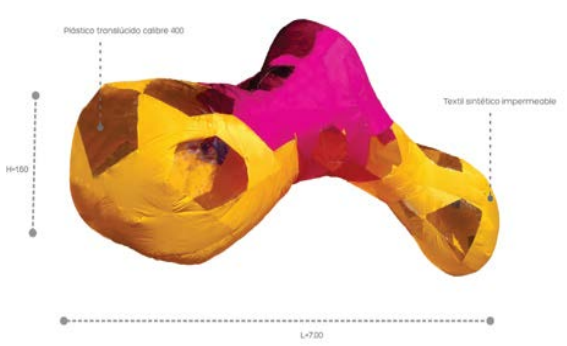

Figura 15: Prototipo escala 1:1 


\section{INSTRUMENTOS DE MEDICIÓN}

La actividad desarrollada durante la implementación del artefacto será medida y registrada por medio de diversas herramientas tanto manuales como digitales, para lo cual se implementa el uso de conteo de afluencia, registro fotográfico y en video, encuestas y recolección de datos a través de formatos digitales que pueden ser geo referenciados, con la finalidad de obtener una base de datos; de esta forma la célula se alimenta de la actividad e información proporcionada por los habitantes de la comunidad, esto la informa y le da vida al objeto inerte; finalmente este grupo de datos puede ser almacenado en un mapa digital, el cual irá ubicando los puntos intervenidos y generando una red de canchas subutilizadas re activadas por medio del artefacto.

Para llevar un control de la comparación de datos, estos se recaban antes y después de la intervención lo que permite contrastarlos y evaluar el impacto que se tuvo con la instalación, posteriormente es pertinente evaluar una serie de reflexiones en torno a cómo este tipo de artefactos pueden impulsar el deseo de participación y colaboración comunitaria, así como el poder que tienen para generar un sentido de empoderamiento social independiente a las acciones que tienen las políticas gubernamentales en el espacio público de la ciudad.

Tabla 4: Recolección de información de la célula transformación de su espacio público, haciéndolo consciente de su rol vital en la revitalización del mismo.

"El capitalismo, no muy diferente de la esquizofrenia, se define por un proceso de decodificación $y$ desterritorialización de todos los códigos y territorios de poder tradicionales, y desata dinámicas sociales sin precedentes" (Deleuze et al., 2015).

\section{CONCLUSIONES}

Las transformaciones que han sufrido las ciudades a lo largo de los años han provocado que muchos de los procesos de diseño queden obsoletos y ya no respondan a las necesidades actuales de la población, las cuáles se ven más incentivadas a realizar sus propias intervenciones y tomar el territorio en sus manos, aunado a esto el surgimiento de nuevas tecnologías hacen posible la visualización de nuevos territorios dentro del diseño, lo cual empuja al espacio público a nuevos límites.

La ciudad actual está en constante cambio por lo que se ve obligada a incorporar procesos y herramientas coherentes con las condiciones de transformación y

\begin{tabular}{|c|c|c|c|}
\hline \multicolumn{4}{|c|}{ Alimentación de la célula } \\
\hline Indicadores a medir & Instrumento & Tiempo de medición & Formato de recolección \\
\hline Estado físico del sitio & Cámara digital & $\begin{array}{l}\text { Antes y durante la } \\
\text { intervención }\end{array}$ & $\begin{array}{l}\text { Fotografía } \\
\text { georreferenciada }\end{array}$ \\
\hline Tipos y conteo de actores & $\begin{array}{l}\text { Epicollect (aplicación digital } \\
\text { de recolección de datos) }\end{array}$ & $\begin{array}{l}2 \text { hrs (duración de } \\
\text { intervención) }\end{array}$ & $\begin{array}{l}\text { Formato digital de } \\
\text { recolección de datos }\end{array}$ \\
\hline Tiempo de permanencia & Cronómetro & $\begin{array}{l}\text { Min. } 5 \text { minutos y Max. } 15 \\
\text { minutos en adelante }\end{array}$ & $\begin{array}{l}\text { Formato digital de } \\
\text { recolección de datos }\end{array}$ \\
\hline Grado de configuración del espacio & Video y fotografías & $\begin{array}{l}2 \text { hrs (duración de } \\
\text { intervención) }\end{array}$ & $\begin{array}{l}\text { Mapeo de posición sobre } \\
\text { fotografías y video }\end{array}$ \\
\hline $\begin{array}{l}\text { Grado de interacción entre el artefacto y } \\
\text { el sitio }\end{array}$ & $\begin{array}{l}\text { Cantidad de activadores } \\
\text { utilizados }\end{array}$ & $\begin{array}{l}2 \text { hrs (duración de } \\
\text { intervención) }\end{array}$ & $\begin{array}{l}\text { Formato digital de } \\
\text { recolección de datos }\end{array}$ \\
\hline índice de atracción & $\begin{array}{l}\text { Conteo manual de } \\
\text { participantes }\end{array}$ & $\begin{array}{l}2 \text { hrs (duración de } \\
\text { intervención) }\end{array}$ & $\begin{array}{l}\text { Formato digital de } \\
\text { recolección de datos }\end{array}$ \\
\hline
\end{tabular}

\section{DISCUSIÓN}

De acuerdo al trabajo urbano-sociológico realizado por las arquitectas españolas Isabel Gutierrez y Candela Morado referente a lo que ellas llaman "Microdispositivos urbanos" («activistark», s. f.) estos surgen como una respuesta a la evolución social de las ciudades y al despertar de verse relegados como una parte fundamental del diseño de sus espacios públicos a lo largo de la historia.

Por lo que el artefacto urbano es un elemento que convierte al usuario en actor y participante activo de la regeneración, las estructuras vivas, efímeras y móviles son un reflejo de las necesidades actuales. Los "microdispositivos", artefactos o intervenciones temporales incluyen estrategias de visibilidad y transparencia de procesos, así como una alternativa para intervenir en el diseño cooperativo "desde abajo" («activistark», s. f.).

Estas intervenciones también tienen el poder de explorar nuevos límites creativos en lo referente a los materiales de diseño y su aplicación, ya que al ser temporales pueden 
probar la resistencia y comportamiento de materiales como las membranas plásticas o los textiles cuya aplicación abre nuevas posibilidades técnicas y espaciales permitiendo generar procesos y ambientes más flexibles. (Toomey, 2006).

En una sutil reflexión sobre el sentido del artefacto, Tharp (Tharp \& Tharp, 2019) aborda este, desde una perspectiva argumentativa afirmando que los diseñadores crean artefactos que implícita o explícitamente comunican y valoran el escenario que los circunscribe. El artefacto en este contexto a veces ocupa un lugar central a veces, tiene un papel secundario. Estos escenarios son visiones de mundos más profundos de prácticas y valores socioculturales, ya sean previstos o imprevistos, ya sean fácticos o ficticios. A través de los cuales la reflexión del encuentro de los habitantes sobre este esquema del mundo, emerge el mensaje del diseñador. Los artefactos en última instancia engendran discurso.

De la misma manera Tharp (Tharp \& Tharp, 2019), advierte que la potencia particular que tienen los artefactos, y en general las artes visuales para que en cierto nivel y medida se pueda visualizar lo que el lenguaje solo puede describir. De esta manera es que los diseñadores pueden hacer que las ideas sean tangibles y experimentales

En este sentido la exploración del espacio público mediante estructuras neumáticas permite salir de los ejes convencionales de lo físico y lo perceptual, provocando a modificar el concepto entre la construcción del espacio público y la capacidad que tienen todos los ciudadanos de intervenirlo con acciones pequeñas pero poderosas.

Cuando Virilio alude el sentido del espacio arquitectónico en la geometría derivada de los vectores espacio temporales, influye para que en este ejercicio experimental se establezca una nueva estética buscando que esta sea una máquina de comunicación, un artefacto transferencia o trasmisión de conocimiento y sensaciones (Virilio, 2003)

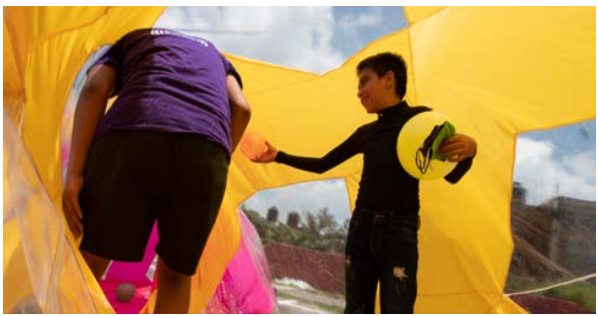

Figura 16: Experimentación de prototipo en sitio

"To unfold, inflate and see each other in a black and white red purple cloud balloon can (conditions right) help to break down people's category walls about each other and their own abilities and can be a hint at the idea that maybe, maybe anybody can, should, must take space-making beautifying into her, his, own hands." (Ant Farm, 1973)

\section{AGRADECIMENTOS}

Nos complace agradecer al Comité Ejecutivo Internacional de SIGraDi por la inclusión y difusión de la presente investigación como parte del congreso del año 2020, así como a la Universidad Michoacana de San Nicolás de Hidalgo, sede de la Maestría en Diseño Avanzado, la cuál permitió el desarrollo del tema por medio de la guía y colaboración tanto del cuerpo académico como de las herramientas facilitadas durante el posgrado. De igual manera se extiende el agradecimiento al Consejo Nacional de Ciencia y Tecnología (CONACYT) cuyo apoyo económico ha permitido el financiamiento de este trabajo.

\section{REFERENCIAS}

activistark::: GENEALOGIA DE MICRO-DISPOSITIVOS URBANOS:: Una perspectiva antropologica para la deconstruccion de los proyectos ciudadanos emergentes :.:: por Isabel Gutierrez y Candela Morado. (s. f.). activistark. Recuperado 13 de marzo de 2020, de https://activistark.blogspot.com/2014/01/genealogia-de-microdispositivos.html

Ant Farm. (1973). Inflatocookbook. Ant Corps.

Borja, J., Universitat Autónoma de Barcelona (España), \& Institut de Ciences Politiques i Socials. (2000). Ciudad y ciudadanía: Dos notas. ICPS.

Deleuze, G., Guattari, F., \& Vázquez Pérez José. (2015). Mil mesetas: Capitalismo y esquizofrenia. Pre-Textos.

Ezio Manzini: La innovación social propone un nuevo territorio para el diseño. (2012, abril 11). Di-Conexiones. http://www.diconexiones.com/ezio-manzini-la-innovacion-social-propone-unnuevo-territorio-para-el-diseno/

Gehl, J., \& Gemzøe, L. (2002). Nuevos espacios urbanos. Gustavo Gili.

Manu, A. (1998). Tool Toys: Tools with an Element of Play. Danish Design Center.

Manzini, E., \& Vega Pindado, E. (2015). Cuando todos diseñan: Una introducción al diseño para la innovación social. Experimenta.

Spiller, N. (2011). Protocell architecture. Wiley, J.

Tharp, B. M., \& Tharp, S. M. (2019). Discursive Design: Critical, Speculative, and Alternative Things (K. Friedman \& E. Stolterman, Eds.). Mit Press.

Toomey, A. (2006). Architextiles. AD Architectural Design, 76(6), 80-81.

Virilio, P. (2003). Estética de la desaparición. Editorial Anagrama.

Wahl, D. C. (2016). Designing Regenerative Cultures. Triarchy Press Lt. 